\title{
Alcohol breaks down interhemispheric inhibition in females but not in males
}

\author{
Alcohol and frontal connectivity
}

\author{
Sylco S. Hoppenbrouwers • Dennis Hofman •
}

Dennis J. L. G. Schutter

Received: 8 October 2009 /Accepted: 25 November 2009 /Published online: 18 December 2009

(C) The Author(s) 2009. This article is published with open access at Springerlink.com

\begin{abstract}
Introduction Alcohol has renowned behavioral disinhibitory properties which are suggested to involve reductions in frontal lobe functioning as a result of diminished interhemispheric connectivity.

Methods To examine sex differences in frontal interhemispheric connectivity in response to alcohol, 12 female and ten male healthy volunteers received a single administration of $0.5 \%$ alcohol in a placebo-controlled counterbalanced crossover design. Paired-pulse transcranial magnetic stimulation was applied to measure transcallosal inhibition (TCI) between the left and right primary motor cortex (M1). Results Results showed significant reductions in TCI after alcohol administration in female participants exclusively. Discussion These findings provide the first evidence that moderate doses of alcohol differentially affect frontal interhemispheric connectivity in males and females. The present data may shed new light on the physiological mechanisms underlying sex differences in the susceptibility to alcohol.
\end{abstract}

Keywords Alcohol · Frontal cortex ·

Interhemispheric connectivity - Transcallosal inhibition .

Transcranial magnetic stimulation

S. S. Hoppenbrouwers $(\bowtie) \cdot$ D. Hofman • D. J. L. G. Schutter

Department of Experimental Psychology,

Helmholtz Research Institute, Utrecht University,

Heidelberglaan 2,

3584 CS Utrecht, The Netherlands

e-mail: s.s.hoppenbrouwers@uu.nl

\section{Introduction}

In the last decades, alcohol consumption steadily increased culminating in 3.2 million yearly deaths either as a direct or indirect result of alcohol worldwide (World Health Organisation 2004). Moderate doses of alcohol $(\leq 0.5 \%)$ can produce feelings of relaxation and release of response inhibition, whereas higher doses of alcohol (1-1.5\%) cause impairments in motor coordination and vision. Extremely high alcohol levels $(\geq 2.5 \%$ ) ultimately lead to coma and death (Hieda et al. 2005). Interestingly, the effects of alcohol seem to be more pronounced in females than in males (Mills and Bisgrove 1983; Niaura et al. 1987; Mumenthaler et al. 1999; Hommer 2003). Sex differences in response to alcohol intake can in part be explained by differences in the percentage of body fat (Addolorato et al. 1999) and the availability of the enzyme alcohol dehydrogenase which breaks down alcohol (Baraona et al. 2001). However, other research suggests that females differ from males on the innate physiological susceptibility to the central effects of alcohol (Hommer et al. 1996, 2001; Agartz et al. 2003; Sasaki et al. 2009). For instance, chronic misuse of alcohol leaves female alcoholics with more brain damage than their male counterparts (Hommer et al. 2001; Hommer 2003). Notably, however, moderate drinking habits also affect the female brain more strongly than the male brain (Sasaki et al. 2009).

In addition to the general effects on the central and peripheral nervous system, alcohol typically affects frontal cortical functions such as response inhibition and cognitive regulation (Jentsch and Taylor 1999; Lyvers 2000). In fact, even though alcohol diffuses through all biological membranes and is distributed throughout the body, the anterior cortical regions of the brain have been shown to be especially vulnerable to the acute (Weitlauf and Woodward 
2008) and chronic (Wobrock et al. 2009) effects of alcohol. Low doses of alcohol already reduce excitability of the frontal cortex as evidenced by transcranial magnetic stimulation (TMS) and electroencephalography (EEG) (Ziemann et al. 1995; Kähkönen et al. 2001, 2003). This reduction in frontal cortical excitability has been argued to relate to binding of alcohol to gamma-amino butyric acid (GABA)-a receptors in addition to alcohol-related inhibition of $N$-methyl D-aspartate (NMDA) currents (Kähkönen et al. 2003). However, the precise mechanisms that underlie the behavioral effects of alcohol and possible sex differences remain unclear. One possible mechanism may involve (transient) frontal lobe impairments arguably due to reductions in effective frontal interhemispheric connectivity (Kähkönen et al. 2001). Frontal interhemispheric connectivity refers to callosal signal transfer between the left and right frontal cortex (Aboitiz and Montiel 2003). This signal transfer can be measured with paired-pulse TMS wherein the connectivity between the left and right primary motor cortex (M1) is operationalized by applying a conditioning pulse to ipsilateral M1 followed by a test pulse to contralateral M1 $10 \mathrm{~ms}$ later (Ferbert et al. 1992). Compared to the motor evoked potential (MEP) elicited by the test pulse alone, the conditioning pulse inhibits the MEP of the test pulse by about 50\% (Daskalakis et al. 2002). This so-called transcallosal inhibition (TCI) reflects an important physiological mechanism by which the frontal lobes interact and contribute to behavior by providing a noninvasive way to study frontal interhemispheric connectivity (Hofman and Schutter 2009).

The aim of the present placebo-controlled counterbalanced cross-over design was to examine sex differences in frontal interhemispheric connectivity in response to alcohol intake as indexed by TCI. We hypothesized that alcohol will reduce TCI in both males and females. In addition, it is expected that reductions in TCI will be more pronounced in females than in males.

\section{Materials and methods}

\section{Participants}

Twenty-two healthy volunteers (ten males, mean $\pm \mathrm{SD}$, $22.5 \pm 0.85$ years of age) participated in this study. All participants were right-handed, nonsmoking, and free of any psychiatric or neurological disorders and had more than 12 years of education. All female participants used oral contraceptives. Subjects had no history of alcohol abuse or dependence as measured with the Alcohol Use Disorder Identification Test (AUDIT; Berks and McCormick 2008). Written informed consent was obtained, and volunteers were paid for participation. The study was approved by the medical ethical committee of the University Medical Centre Utrecht and in accordance with the Declaration of Helsinki. All participants were naïve to the aim of the study.

Alcohol administration and monitoring

The amount of alcohol which would induce a blood alcohol concentration (BAC) of $0.5 \%$ was estimated with Widmark's formula:

grams of pure alcohol

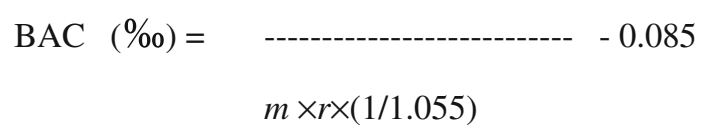

where $m$ is body weight and $r$ refers to the distribution ratio which for men is on average 0.68 and for women 0.55 (Friel et al. 1995). The alcoholic solution $(300 \mathrm{ml})$ consisted of alcohol mixed with orange juice and a few drops of peppermint oil to mask the alcohol. Placebo $(300 \mathrm{ml})$ consisted of orange juice and peppermint oil. Participants' BAC was measured every 5 min using an AlcoMate CORE AL-600 Pro Alcohol Breath Analyzer (AK Solutions USA LLC, Palisades Park, NJ, USA). Measurements were started when BAC approximated $0.5 \%$. Importantly, TCI was measured at the start of the downward limb of the BAC curve because at this point, BAC values are suggested to be relatively stable (Verster et al. 2002).

\section{Transcranial magnetic stimulation}

TMS was performed using two biphasic magnetic brain stimulators (maximum output 4,160 A peak/1,750 VAC peak) and an iron core coil (Neotonus, Atlanta, GA, USA) over the left and right primary motor cortex (M1). Bilateral paired-pulse TMS was applied to measure TCI. In this paradigm, the MEP following the single test pulse (unconditioned MEP, uMEP) to M1 is compared to the MEP evoked by the test pulse that is preceded by a conditioning pulse to the contralateral M1 $10 \mathrm{~ms}$ earlier (conditioned MEP, cMEP; Ferbert et al. 1992). The mean percentage reduction between the unconditioned and conditioned MEP was calculated. Stimulation intensity was set at $120 \%$ of the individual motor threshold (MT). To minimize anticipation effects, TMS was applied at a frequency of $0.18-0.2 \mathrm{~Hz}$. The following conditions were applied in random counterbalanced order: (1) a single test pulse to the left M1 ( $\left.\mathrm{uMEP}_{\text {left }}\right)$, (2) a conditioning pulse to the right $\mathrm{M} 1$ followed by a test pulse to the left $\mathrm{M} 1$ ( $\left.\mathrm{cMEP}_{\text {left }}\right)$, (3) a single test pulse to the right M1 (uMEP right), and (4) a conditioning pulse to the left $\mathrm{M} 1$ followed by a test pulse to the right M1 (cMEP ${ }_{\text {right }}$; Ferbert et al. 1992). 


\section{Electromyographic recordings}

The electromyogram (EMG) was recorded using sintered $11 \times 17$-mm active $\mathrm{Ag}-\mathrm{AgCl}$ electrodes with the ActiveTwo system (BioSemi, Amsterdam, The Netherlands) relative to the common mode sense in a belly-tendon arrangement. The MEP was recorded from the left and right abductor pollicis brevis (APB). The active electrode was placed over the muscle belly of the APB. The reference electrode was placed over the proximal phalanx of the thumb. The ground electrode was attached to the wrist. EMG signal was digitized at $16 \mathrm{kHz}$, low-pass filtered $(-3 \mathrm{~dB}$ cutoff frequency $3,334 \mathrm{~Hz}$; roll-off $30 \mathrm{~dB} /$ octave), and offline high-pass filtered ( $-3 \mathrm{~dB}$ cutoff frequency $20 \mathrm{~Hz}$; roll-off $48 \mathrm{~dB} /$ octave).

\section{Procedure}

In the present crossover design, participants received either a single dose of alcohol or placebo on one of the two occasions in a randomized counterbalanced order. Prior to the testing sessions and on a separate day, participants were invited upon the laboratory. A safety-screening list was administered to check for contraindications to TMS (Keel et al. 2001), and health was checked with a standard interview. In addition, safety issues and experimental procedures were explained to the subject, and written informed consent was obtained. Alcohol abuse and alcohol dependence were indexed with the AUDIT (Berner et al. 2007), and participants with AUDIT scores of 0 or $>8$ were excluded. Right handedness was assessed with the Edinburgh handedness inventory (Oldfield 1971). Resting MT was determined using the standardized motor threshold estimation procedure (Schutter and van Honk 2006; see Table 1 for details).

Participants were instructed to refrain from consuming alcoholic beverages $24 \mathrm{~h}$ prior to the testing session, not to consume coffee, tea, or chocolate $5 \mathrm{~h}$ before testing and to use their last meal at least $2 \mathrm{~h}$ prior to the testing session. To control for circadian rhythms, both testing sessions were conducted at the same time of the day. Each testing session

Table 1 Main demographics and characteristics of the participants

\begin{tabular}{lrrr}
\hline & \multicolumn{1}{l}{$\begin{array}{l}\text { Males } \\
(\text { mean } \pm \text { SEM) }\end{array}$} & \multicolumn{1}{l}{$\begin{array}{l}\text { Females } \\
(\text { mean } \pm \text { SEM) }\end{array}$} & \multicolumn{1}{l}{$p$} \\
\hline Age (in years) & $23.3 \pm 1.4$ & $21.9 \pm 1.1$ & 0.43 \\
AUDIT & $6.4 \pm 0.6$ & $5.8 \pm 0.6$ & 0.53 \\
MT left hemisphere & $47.5 \pm 2.8$ & $49 \pm 2.0$ & 0.66 \\
MT right hemisphere & $45.2 \pm 2.7$ & $47.8 \pm 2.2$ & 0.47 \\
Handedness & $45.5 \pm 0.7$ & $46.3 \pm 0.7$ & 0.44 \\
\hline
\end{tabular}

MT motor threshold was separated by at least $24 \mathrm{~h}$. Moreover, to minimize the effects of additional hormonal influences, female participants were not tested during the stopping week.

Each experimental session started with a BAC measurement to ascertain sobriety after which participants consumed the alcoholic solution or placebo. Participants had 2 min to drink the solution while keeping their nose closed with their hand. The time between administration and paired-pulse TMS was approximately $30 \mathrm{~min}$. During this period, EMG electrodes were attached to the left and right $\mathrm{APB}$, and the optimal target sites for TMS were determined. TCI was measured when the BAC curve approximated $0.5 \%$ on the descending limb of the BAC curve (Verster et al. 2002). At the end of the final session, participants were debriefed and paid for participation. Both testing sessions took approximately $1 \mathrm{~h}$ to complete.

Data reduction and analysis

MEP amplitude was quantified as the peak-to-peak amplitude of the maximal EMG response. Left-to-right (1-rTCI) and right-to-left transcallosal inhibition (r-lTCI) were expressed according to the formulas $\left[\left(1-\left(\mathrm{cMEP}_{\text {right }} / \mathrm{uMEP}_{\text {right }}\right)\right) \times 100\right]$ and $\left[\left(1-\left(\mathrm{cMEP}_{\text {left }} / \mathrm{uMEP}_{\text {left }}\right) \times 100\right]\right.$, respectively.

A general linear model (GLM) for repeated measurements with drug (alcohol versus placebo) and TCI (left to right versus right to left) as within-subjects factor and sex (female and male) as a between-subjects factor was run to examine the alcohol-related effects and possible sex differences on TCI. To rule out possible alcohol-related effects of single-pulse TMS on TCI, an additional GLM for repeated measurements with drug (alcohol versus placebo) and side (left versus right hemisphere) as within-subjects factors and sex (female versus male) as a between-subjects factor was performed. Finally, to exclude effects of baseline EMG activity on TCI, a $2 \times 2$ GLM for repeated measurements with drug (alcohol versus placebo) and EMG (left versus right hand) as within-subjects factor and sex (female versus male) as within-subjects factor was conducted. The alpha level of significance was set at 0.05 (two-tailed).

\section{Results}

$\mathrm{BAC}$ values did not differ between males (mean $\pm \mathrm{SD}, 0.69 \pm$ 0.04 ) and females (mean $\pm \mathrm{SD}, 0.603 \pm 0.05 ; p=0.17$ ). Our hypothesis that alcohol would reduce TCI in both males and females was not confirmed $(F(1,21)=2,752, p=0.113)$. In contrast, a significant drug $\times$ sex interaction $(F(1,21)=$ $4.95 ; p=0.038)$ was observed. Post hoc analyses demonstrated that alcohol significantly reduced TCI in females $\left(F(1,11)=12.52 ; p=0.005 ; \eta_{\mathrm{p}}{ }^{2}=0.53\right)$ but not in males $(F(1,9)=0.10 ; p=0.76$; Fig. 1$)$. 


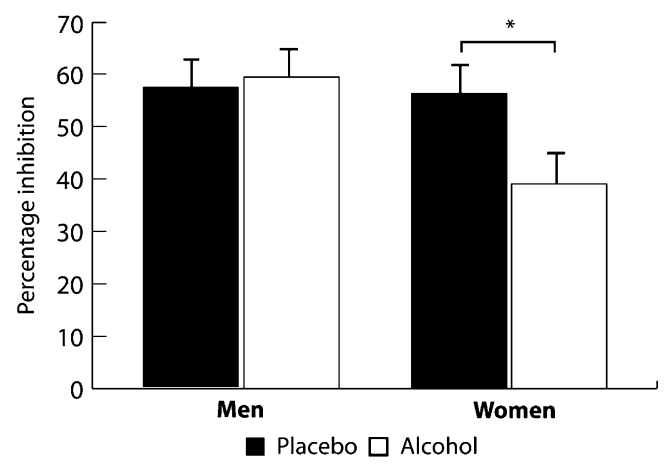

Fig. 1 Mean and standard error of the mean of frontal interhemispheric inhibition in the placebo and alcohol condition

The observed sex difference in TCI after alcohol could not be explained by reductions in single-pulse TMS as demonstrated by a nonsignificant drug $\times$ sex interaction $(F$ $(1,21)=1.024 ; p=0.324)$. However, a main effect of alcohol on single-pulse TMS $(F(1,21)=4,517 ; p=0.046$; $\eta_{\mathrm{p}}{ }^{2}=0.184$ ) was observed, replicating prior studies that found alcohol-related reductions in cortical excitability (Ziemann et al. 1995; Kähkönen et al. 2003). Finally, the sex-related effect of alcohol on TCI could not be explained by differences in baseline EMG activity as shown by the nonsignificant drug $\times$ sex interaction $(F(1,21)=0.278 ; p=$ 0.604). No main effects of alcohol on baseline EMG was observed $(F(1,21)=0.270 ; p=0.609)$. For an impression of the raw data, please see Table 2 .

\section{Discussion}

The aim of the present study was to examine alcoholrelated sex differences in frontal interhemispheric connectivity and found reductions in frontal interhemispheric connectivity to a moderate dose of alcohol in healthy female but not male subjects. To the best of our knowledge, this is the first study to demonstrate evidence for sex differences in frontal interhemispheric connectivity in response to alcohol that cannot be explained by effects of alcohol on baseline EMG activity or single-pulse TMS. The global reduction in cortical excitability as evidenced by a decrease in MEP size in both sexes replicates earlier findings by Ziemann et al. (1995) and Kähkönen et al. (2003). This reduction in frontal cortical excitability has been explained in terms of increased binding of alcohol to GABA-a receptors and alcohol-related inhibition of NMDA currents (Kähkönen et al. 2003). In measuring TCI with paired-pulse TMS, the conditioning pulse administered over the motor cortex is thought to activate glutamatergic excitatory callosal fibers terminating on local GABAergic interneurons of the contralateral motor cortex (Daskalakis et al. 2002). Excitation of these GABAergic inhibitory interneurons results in inhibition of the pyramidal motor neurons which causes a reduction in the MEP size. Consequently, a decrease in frontal interhemispheric connectivity may relate to the influence of alcohol on either NMDA glutamate receptor functioning or GABAergic inhibitory interneurons. Previous TMS research has demonstrated that alcohol mainly influences GABAergic functioning leaving glutamatergic excitatory transmission relatively unaffected (Ziemann et al. 1995; Conte et al. 2008). In contrast, alcohol has also been shown to attenuate glutamate/NMDA receptor functioning (Lovinger et al. 1989; White et al. 1990; Masood et al. 1994; Weitlauf and Woodward 2008) leaving open the possibility that alcohol may have disrupted the excitatory callosal pathway that terminates on inhibitory interneurons. This would result in less excitation of inhibitory interneurons, subsequently leading to a relatively increased MEP size. However, the crucial point revolves around the question how to explain the alcohol-related sex difference on frontal interhemispheric connectivity, and at present, the precise physiological mechanisms through which alcohol reduces frontal interhemispheric connectivity in females remain to be elucidated.

A possible role for steroid hormones in explaining the presently observed sex differences is evidenced by a study in which testosterone was found to counteracts some of the effects of alcohol (Khalil et al. 2005). In this study, testosterone was shown to diminish alcohol-induced deficits in spatial memory (Khalil et al. 2005). Interestingly,

Table 2 Sex-related effect of alcohol on TCI, single pulse, and EMG

\begin{tabular}{|c|c|c|c|c|c|c|}
\hline & \multicolumn{2}{|l|}{ TCI } & \multicolumn{2}{|l|}{ Single pulse } & \multicolumn{2}{|l|}{ EMG } \\
\hline & Males & Females & Males & Females & Males & Females \\
\hline Placebo $($ mean \pm SEM) & $57.1 \pm 5.9$ & $56.3 \pm 7.2$ & $1,843.2 \pm 235.6$ & $1,880.8 \pm 653.8$ & $4,769 \pm 704.9$ & $8,574.7 \pm 2,403.2$ \\
\hline Alcohol $($ mean \pm SEM) & $59.4 \pm 7.2$ & $40.7 \pm 8.1$ & $1,573.2 \pm 336$ & $1,119.8 \pm 396.7$ & $6,351.9 \pm 1,118.2$ & $8,563.9 \pm 1,256.4$ \\
\hline
\end{tabular}

Single-pulse TMS is expressed in microvolt

$T C I$ transcallosal inhibition (expressed in percentage inhibition of single-pulse TMS), EMG electromyogram (expressed in microvolt), SEM standard error of the mean 
acute alcohol administration reduces testosterone production in both males and females (Ylikahri et al. 1980; Valimaki et al. 1984). Together with the notion that men have multiple times more testosterone than females, testosterone may play a role in the currently observed reductions of TCI in females exclusively. In agreement, we recently found evidence that a single administration of testosterone significantly increases frontal interhemispheric connectivity in healthy female subjects (Hoppenbrouwers et al., submitted). In sum, even though sex differences in steroid hormones may provide for an explanation of our findings, it remains elusive whether these sex differences already surface after an acute administration of alcohol. It is suggested that the decrease in frontal interhemispheric connectivity observed in females will most likely involve complex interactions between steroid hormones and the combined action of alcohol on GABAergic interneurons and NMDA glutamate receptor functioning in the corpus callosum.

Despite the observed female vulnerability to the effects of alcohol on frontal interhemispheric connectivity, several issues should be mentioned. First, the relationship between alcohol-related effects on frontal interhemispheric connectivity and behavior is of importance. Executive functions including behavioral inhibition and cognitive regulation are normally ascribed to the prefrontal cortex (PFC) rather than M1. Even though both M1 and PFC are part of the frontal cortex, our current findings on M1 interhemispheric connectivity cannot simply be extrapolated to the PFC. Defensibly, there is evidence from recent interleaved TMSEEG studies showing that M1 and the PFC share similar physiological properties (Daskalakis et al. 2008). Second, the fact that we observed reductions in frontal interhemispheric to alcohol consumption in females only does not in any way imply that men are immune to the effects of alcohol. In other words, males will likely demonstrate similar reductions in frontal interhemispheric connectivity at higher BAC levels. Third, in our sample, all female participants were taking oral contraceptives to abolish fluctuations in steroid levels at the time of testing. However, oral contraceptives have been shown to reduce plasma levels of progesterone metabolites in female rats and women (Follesa et al. 2001). Some of these metabolites (e.g., allopregnanolone) are highly potent endogenous positive modulators of the GABA-a receptor (Mitchell et al. 2008). It has been suggested that the combined effects of endogenous steroids and alcohol modulate GABA-a receptor functioning (Follesa et al. 2004). In contrast, alcohol has also been shown to increase allopregnanolone levels in the cerebral cortex (VanDoren et al. 2000), an increase that has proven effective to potentiate GABA-a receptor-mediated inhibition in the brain (VanDoren et al. 2000) and explain some of the physiological and behavioral effects of alcohol (VanDoren et al. 2000; Izumi et al. 2007). Taken together, these findings suggest that the contraceptive- and alcoholrelated increases in allopregnanolone levels may have contributed to the presently observed reductions in TCI. As it stands, the present findings can only be generalized to males and females taking oral contraceptives. Finally, an additional line of evidence consisting of conventional TMS measures of GABA function such as short-interval intracortical inhibition and long-interval intracortical cortical inhibition could potentially has given more direct evidence for the exact mechanisms underlying the observed effect.

In conclusion, the present study provides the first evidence for reductions in frontal interhemispheric connectivity in females but not in males following a moderate dose of alcohol. Future research may focus on alcohol doseresponse patterns in males and the involvement of steroid hormones to further explain the observed sex differences in interhemispheric connectivity. In addition, to clarify the potential role of contraceptives, future research may also look into the effects of alcohol on interhemispheric inhibition in females not using contraceptives.

Acknowledgments This work was supported by an Innovational Research Grant (VIDI 452-07-012) from the Netherlands Organization for Scientific Research (NWO).

Open Access This article is distributed under the terms of the Creative Commons Attribution Noncommercial License which permits any noncommercial use, distribution, and reproduction in any medium, provided the original author(s) and source are credited.

\section{References}

Aboitiz F, Montiel J (2003) One hundred million years of interhemispheric communication: the history of the corpus callosum. Braz J Med Biol Res 36(4):409-420

Addolorato G, Capristo E et al (1999) Nutritional status and body fluid distribution in chronic alcoholics compared with controls. Alcohol Clin Exp Res 23(7):1232-1237

Agartz I, Shoaf S et al (2003) CSF monoamine metabolites and MRI brain volumes in alcohol dependence. Psychiatry Res 122(1):21-35

Baraona E, Abittan CS et al (2001) Gender differences in pharmacokinetics of alcohol. Alcohol Clin Exp Res 25(4):502-507

Berks J, McCormick R (2008) Screening for alcohol misuse in elderly primary care patients: a systematic literature review. Int Psychogeriatr 20(6):1090-1103

Berner MM, Kriston L et al (2007) The alcohol use disorders identification test for detecting at-risk drinking: a systematic review and meta-analysis. J Stud Alcohol Drugs 68(3):461-473

Conte A, Attilia ML et al (2008) Acute and chronic effects of ethanol on cortical excitability. Clin Neurophysiol 119(3):667-674

Daskalakis ZJ, Christensen BK et al (2002) The mechanisms of interhemispheric inhibition in the human motor cortex. J Physiol 543(Pt 1):317-326

Daskalakis ZJ, Farzan F et al (2008) Long-interval cortical inhibition from the dorsolateral prefrontal cortex: a TMS-EEG study. Neuropsychopharmacology 33:2860-2869

Ferbert A, Priori A et al (1992) Interhemispheric inhibition of the human motor cortex. J Physiol 453:525-546 
Follesa P, Concas A et al (2001) Role of allopregnanolone in regulation of $\mathrm{GABA}(\mathrm{A})$ receptor plasticity during long-term exposure to and withdrawal from progesterone. Brain Res Brain Res Rev 37(1-3):81-90

Follesa P, Biggio F et al (2004) Modulation of GABA(A) receptor gene expression by allopregnanolone and ethanol. Eur $\mathrm{J}$ Pharmacol 500(1-3):413-425

Friel PN, Baer JS et al (1995) Variability of ethanol absorption and breath concentrations during a large-scale alcohol administration study. Alcohol Clin Exp Res 19(4):1055-1060

Hieda Y, Takeshita $\mathrm{H}$ et al (2005) A fatal case of pure ethanol ingestion. Forensic Sci Int 149(2-3):243-247

Hofman D, Schutter DJ (2009) Inside the wire: aggression and functional interhemispheric connectivity in the human brain. Psychophysiology 46:1054-1058

Hommer DW (2003) Male and female sensitivity to alcohol-induced brain damage. Alcohol Res Health 27(2):181-185

Hommer D, Momenan R et al (1996) Decreased corpus callosum size among alcoholic women. Arch Neurol 53(4):359-363

Hommer D, Momenan R et al (2001) Evidence for a gender-related effect of alcoholism on brain volumes. Am J Psychiatry 158 (2): 198-204

Izumi Y, Murayama K et al (2007) GABAergic neurosteroids mediate the effects of ethanol on long-term potentiation in rat hippocampal slices. Eur J NeuroSci 26(7):1881-1888

Jentsch JD, Taylor JR (1999) Impulsivity resulting from frontostriatal dysfunction in drug abuse: implications for the control of behavior by reward-related stimuli. Psychopharmacology (Berl) 146(4):373-390

Kähkönen S, Kesaniemi M et al (2001) Ethanol modulates cortical activity: direct evidence with combined TMS and EEG. Neuroimage 14(2):322-328

Kähkönen S, Wilenius $J$ et al (2003) Alcohol reduces prefrontal cortical excitability in humans: a combined TMS and EEG study. Neuropsychopharmacology 28(4):747-754

Keel JC, Smith MJ et al (2001) A safety screening questionnaire for transcranial magnetic stimulation. Clin Neurophysiol 112(4):720

Khalil R, King MA et al (2005) Testosterone reverses ethanol-induced deficit in spatial reference memory in castrated rats. Pharmacology 75(2):87-92

Lovinger DM, White G et al (1989) Ethanol inhibits NMDA-activated ion current in hippocampal neurons. Science 243(4899): 1721-1724

Lyvers M (2000) "Loss of control" in alcoholism and drug addiction: a neuroscientific interpretation. Exp Clin Psychopharmacol 8 (2):225-249

Masood K, Wu C et al (1994) Differential ethanol sensitivity of recombinant N-methyl-D-aspartate receptor subunits. Mol Pharmacol 45(2):324-329
Mills KC, Bisgrove EZ (1983) Body sway and divided attention performance under the influence of alcohol: dose-response differences between males and females. Alcohol Clin Exp Res 7(4):393-397

Mitchell EA, Herd MB et al (2008) Neurosteroid modulation of GABAA receptors: molecular determinants and significance in health and disease. Neurochem Int 52(4-5):588-595

Mumenthaler MS, Taylor JL et al (1999) Gender differences in moderate drinking effects. Alcohol Res Health 23(1):55-64

Niaura RS, Nathan PE et al (1987) Gender differences in acute psychomotor, cognitive, and pharmacokinetic response to alcohol. Addict Behav 12(4):345-356

Oldfield RC (1971) The assessment and analysis of handedness: the Edinburgh inventory. Neuropsychologia 9(1):97-113

Sasaki H, Abe O et al (2009) Structural and diffusional brain abnormality related to relatively low level alcohol consumption. Neuroimage 46(2):505-510

Schutter DJLG, van Honk J (2006) A standardized motor threshold estimation procedure for transcranial magnetic stimulation research. J ECT 22(3):176-178

Valimaki MJ, Harkonen M et al (1984) Sex hormones and adrenocortical steroids in men acutely intoxicated with ethanol. Alcohol 1(1):89-93

VanDoren MJ, Matthews DB et al (2000) Neuroactive steroid 3alpha-hydroxy-5alpha-pregnan-20-one modulates electrophysiological and behavioral actions of ethanol. J Neurosci 20(5): 1982-1989

Verster JC, Volkerts ER et al (2002) Residual effects of middle-of-thenight administration of zaleplon and zolpidem on driving ability, memory functions, and psychomotor performance. J Clin Psychopharmacol 22(6):576-583

Weitlauf C, Woodward JJ (2008) Ethanol selectively attenuates NMDAR-mediated synaptic transmission in the prefrontal cortex. Alcohol Clin Exp Res 32(4):690-698

White G, Lovinger DM et al (1990) Ethanol inhibits NMDA-activated current but does not alter GABA-activated current in an isolated adult mammalian neuron. Brain Res 507(2):332-336

Wobrock T, Falkai P et al (2009) Effects of abstinence on brain morphology in alcoholism: a MRI study. Eur Arch Psychiatry Clin Neurosci 259(3):143-150

World Health Organisation (2004) Global status report on alcohol. WHO, Geneva

Ylikahri RH, Huttunen MO et al (1980) Hormonal changes during alcohol intoxication and withdrawal. Pharmacol Biochem Behav 13(Suppl 1):131-137

Ziemann U, Lonnecker S et al (1995) Inhibition of human motor cortex by ethanol. A transcranial magnetic stimulation study. Brain 118(Pt 6):1437-1446 